\title{
Science
}

DAAAS

\section{Plant roots sense soil compaction through restricted ethylene diffusion}

2 Bipin K. Pandey ${ }^{1} \dagger$, Guoqiang Huang ${ }^{2} \dagger$, Rahul Bhosale ${ }^{1}$, Sjon Hartman ${ }^{3,4}$, Craig J. Sturrock ${ }^{1}$,

3 Lottie Jose ${ }^{1}$, Olivier C. Martin ${ }^{5}$, Michal Karady ${ }^{6}$, Laurentius A.C.J. Voesenek ${ }^{3}$, Karin Ljung ${ }^{7}$,

4 Jonathan P. Lynch ${ }^{8}$, Kathleen M. Brown ${ }^{8}$, William R. Whalley ${ }^{9}$, Sacha J. Mooney ${ }^{1}$, Dabing

$5 \quad$ Zhang $^{2 *}$ and Malcolm J. Bennett ${ }^{1 *}$

$6 \uparrow$ Joint First authorship

7 *Corresponding author Emails: malcolm.bennett@nottingham.ac.uk (M.J.B) and

8 zhangdb@sjtu.edu.cn (D.Z)

$9 \quad{ }^{1}$ School of Biosciences, University of Nottingham, Sutton Bonington LE12 5RD, UK

$10 \quad{ }^{2}$ Joint International Research Laboratory of Metabolic \& Developmental Sciences, School of Life

11 Sciences and Biotechnology, Shanghai Jiao Tong University, Shanghai, 200240 China

$12{ }^{3}$ Plant Ecophysiology, Institute of Environmental Biology, Utrecht University, Padualaan 8, 3584

$13 \mathrm{CH}$, Utrecht, Netherlands

$14{ }^{4}$ School of Biosciences, University of Birmingham, B15 2TT, UK

15 5niversities of Paris-Saclay, Paris and Evry, CNRS, INRAE, Institute of Plant Sciences Paris-

16 Saclay (IPS2), Bât. 630, 91192, Gif sur Yvette, France

$17{ }^{6}$ Laboratory of Growth Regulators, Institute of Experimental Botany of the Czech Academy of

18 Sciences and Faculty of Science of Palacký University, Šlechtitelù 27, CZ-78371 Olomouc, Czech

19 Republic

$20{ }^{7}$ Department of Forest Genetics and Plant Physiology, Umeå Plant Science Centre, Swedish

21 University of Agricultural Sciences, Umeå, Sweden 


\section{Science}

MIAAAS

$22{ }^{8}$ Department of Plant Science, The Pennsylvania State University, University Park, PA16802, USA

$23{ }^{9}$ Rothamsted Research, West Common, Harpenden, AL5 2JQ UK

24

25 orcid.org/0000-0002-9614-1347 (BKP)

26 orcid.org/0000-0001-6515-4922 (RB)

27 orcid.org/0000-0002-6709-6436 (SH)

28 orcid.org/0000-0003-0475-390X (MJB)

29 orcid.org/0000-0003-2901-189X (KL)

30 orcid.org/0000-0002-7265-9790 (JPL)

31 orcid.org/0000-0002-4960-5292 (KMB)

32 orcid.org/0000-0002-5603-706X (MK)

33

34 


\section{Science}

DAAAS

\section{Abstract:}

36 Soil compaction represents a major challenge for modern agriculture. Compaction is intuitively

37 thought to reduce root growth by limiting their ability to penetrate harder soils. We report that root

38 growth in compacted soil is instead actively suppressed by the volatile hormone ethylene. Mutant

39 roots insensitive to ethylene penetrate compacted soil more effectively than wildtype. We

40 demonstrate that roots sense mechanical impedance by employing the gaseous signal ethylene, as

41 soil compaction lowers gas diffusion through a reduction in air-filled pores, causing ethylene to

42 accumulate in root tissues and trigger hormone responses that restrict growth. We propose that

43 ethylene acts as an early warning signal for roots to avoid compacted soils, revealing approaches

44 to breed crops resilient to soil compaction.

\section{$45 \quad \mathbf{1 1 8} / \mathbf{1 2 5}$ words}

\section{One Sentence Summary:}

47 Roots sense soil compaction employing the gaseous signal ethylene. 


\section{Science}

DAAAS

Soil compaction impacts global crop cultivation by reducing root penetration in both the upper and deeper soil layers (1). Modern agricultural practices have exacerbated soil compaction,

51 largely due to intensification of operations leading to the deployment of heavier machinery and 52 tillage practices $(2,3)$, severely degrading $\sim 65$ million hectares of land globally (4). Compaction

53 increases soil bulk density and reduces soil porosity, limiting the availability and transport of water 54 and nutrients $(4,5)$. The decrease in soil pore space, especially in large air-filled pores (Fig. 1, Arhizosphere (6). To deal with compacted soils and penetrate cracks, roots are reported to undergo adaptive growth responses, including increasing radial expansion of root tips (1). However, the predominant response of roots is cessation of growth of which the mechanistic basis remains unclear. Here, we report that entrapped ethylene functions as a key signal regulating root growth in compacted soils.

Ethylene is produced by root tissues and its level increases when roots are exposed to compacted soil $(7,8)$. Ethylene concentrations outside the root could increase due to the reduction in soil pore space in compacted soil, impacting gas diffusion from root tissues (Fig 1A-D; figs. S1 and S2). To test this 'restricted gas diffusion' model, we used the EIN3-GFP Arabidopsis ethylene response reporter (9; fig. S3, A-C) and examined the effect of covering root tips with a gas

66 impermeable barrier. In agreement with model assumptions, restricting gas diffusion from root tip

67 tissues triggered a rapid and sustained increase in EIN3-GFP in root elongation zone cell nuclei 68 compared to controls (Fig. 1, F versus E; fig. S3, D-G). This result is consistent with (a) limitation

69 of ethylene release from root tip tissues and (b) changes in gas diffusion rate between roots and 70 the external environment inducing ethylene accumulation and signalling. To rule out that changes 71 in ethylene signalling were related to reduced oxygen levels in root tip tissues, we treated roots 


\section{Science}

72 expressing hypoxia markers pPCO1:GFP-GUS, pPCO2:GFP-GUS (10) and RAP2.12-GFP (11)

73 with the gas impermeable barrier. Hypoxia reporters were not induced by the gas barrier but were

74 induced by submergence (figs. S4 to S6). We conclude that EIN3-GFP induction results from

75 restricted ethylene diffusion, rather than hypoxic conditions (11).

Roots exposed to elevated levels of ethylene exhibit growth inhibition (Fig. 1, I and J)

77 which phenocopies the impact of soil compaction (Fig. 1, G and H). We observed that rice roots

78 grown in $1.1 \mathrm{~g} \mathrm{~cm}^{-3}$ (uncompacted) versus $1.6 \mathrm{~g} \mathrm{~cm}^{-3}$ (compacted) soil bulk densities exhibit

79 reduced root length when exposed to compacted conditions (fig. S7, A and B). Root anatomical analysis revealed that compaction caused a three-fold decrease in epidermal cell length (fig. S7C),

81 matched by a three-fold increase in cortical cell diameter (compare Fig. 1, G and H, and fig. S7D).

82 Similarly, ethylene treatment reduces root length (fig. S8A) whilst increasing root width (Fig. 1, I

83 and J), by decreasing epidermal cell length and increasing cortical cell diameter (fig. S8, B and C).

84 To directly test the functional importance of ethylene during soil compaction, we examined root growth responses of wildtype (WT) rice versus ethylene insensitive mutants osein2 and oseill (12). OsEIN2 (ETHYLENE INSENSITIVE2) encodes a key ethylene signaling component (13).

87 OsEIL1 (EIN3-like 1) encodes a critical transcription factor in the ethylene transduction pathway

88 downstream of OsEIN2 (9). Mutations in rice OsEIN2 and OsEIL1 genes confer ethylene 89 insensitive root elongation phenotypes (12; fig. S9, A and B). To analyse the impact of soil

90 compaction on WT rice versus osein2 root growth, lines were grown in columns either entirely

91 filled with uncompacted soil $\left(1.1 \mathrm{~g} \mathrm{~cm}^{-3}\right)$ or highly compacted soil $\left(1.6 \mathrm{~g} \mathrm{~cm}^{-3}\right.$ with a $1 \mathrm{~cm}$ top layer

92 packed $1.1 \mathrm{~g} \mathrm{~cm}^{-3}$ to help establish seedling root growth). Penetrometer resistance analysis

93 demonstrated that root elongation rate is sensitive to increased soil strength (fig. S10). 


\section{Science}

DAAAS

To quantify the impact of soil compaction on root length of WT versus ethylene mutant

95 lines, we employed the non-invasive X-ray imaging approach, Computed Tomography (CT; Fig.

96 2, A to G). CT imaging revealed that, unlike WT (Fig. 2B), both osein2 and oseill roots were able

97 to penetrate highly compacted soil (Fig. 2, D and F; quantified in Fig. 2G). This result reveals

98 ethylene signalling is critical for triggering root growth responses upon soil compaction.

99 Anatomical analysis of rice mutant roots further demonstrated that under compacted soil

100 conditions, osein2 and oseill root epidermal cells continued to elongate normally, whilst cortical

101 cells did not undergo radial expansion (figs. S11 and S12) compared to WT (fig. S13). Moreover,

102 this growth response also occurs in other classes of roots, since primary and lateral root growth

103 and cortical responses induced by soil compaction are blocked in the ethylene insensitive

104 Arabidopsis mutant etrl (figs. S14 to S17). Similarly, ethylene insensitive mutants in rice (osein2

105 and oseil1) and Arabidopsis (ein3eill) accumulated significantly higher shoot and root biomass in

106 compacted soil conditions compared to WT (figs. S18 and S19). Hence, our rice and Arabidopsis

107 mutant analysis reveals ethylene plays an inhibitory role in both monocot and eudicot root (and

108 shoot) tissues when experiencing soil compaction.

Our results suggest that reduced root growth triggered by soil compaction does not arise

110 from mechanical impedance, but instead represents a timely response controlled by ethylene,

111 perhaps to avoid growth in compacted soils (14). To discriminate between the effects mediated by

112 mechanical impedance versus ethylene, we compared their impact on root tip shape. Soil

113 compaction causes WT rice roots to double in width and their root cap to develop a 'flattened'

114 shape (compare Fig. 2, H and I). Soil compaction-induced radial growth and root cap shape

115 changes were blocked in osein2 (Fig. 2, J and K, and O). Hence, root tip shape changes induced

116 by soil compaction appear to be controlled primarily by ethylene and not by mechanical 


\section{Science}

117 impedance. Indeed, ethylene treatment alone was sufficient to trigger equivalent changes in root

118 width (Fig. 1, I and J, and fig. S8, B and C) and cap shape (Fig. 2, L to N, and fig. S20 similar to 119 roots exposed to soil compaction. Therefore, ethylene represents a critical signal in plants 120 controlling shape changes underpinning root compaction responses.

Given ethylene's functional importance during root responses to compaction, we

122 investigated whether soil mechanical impedance triggered increased ethylene signaling in root

123 tissues. We employed transgenic Arabidopsis and rice either expressing an ethylene biosensor 124 featuring EIN3 (9) or OsEIL1 sequences fused with GFP (fig. S21). In uncompacted soil, 125 35S:EIN3-GFP or proOsEIL1:OsEIL1-GFP reporters in root nuclei were not detectable (Fig. 3, A 126 and D). However, when reporter lines are grown in compacted soil, both ethylene reporters were 127 detected in root elongation zone cells (Fig. 3, B and C, and E). To probe the role of ethylene in 128 other soil types, we grew rice reporter lines in two other soils. Compaction triggered a root ethylene 129 response in clay soil (figs. S21 and S22), and sandy loam soil (Fig. 3E, and fig. S23). Hence, the 130 ethylene-based compaction mechanism appears to operate in different soil types.

How does soil compaction induce elevated ethylene signaling in root tissues? Mechanical

132 impedance could cause roots to upregulate ethylene synthesis. Profiling of the ethylene precursor

133 1-aminocyclopropane-1-carboxylic acid (ACC) in excised rice root tips detected no change in

134 levels after growth in compacted soil versus non-compacted controls (fig. S24). Alternatively, 135 plant roots may sense soil compaction by monitoring ethylene levels. Mathematical modelling 136 predicted slower outward ethylene diffusion rates under compacted soil conditions (Fig. 3H and 137 fig. S25) due to the decreased volume of air-filled pores (1; Fig. 1, A-D and movie S1 and S2).

138 This will result in a higher ethylene concentration close to roots (Fig. 3, F and G) and therefore in 139 root cells, consistent with soil compaction triggering an ethylene response (Fig. 3, B, C and E). 


\section{Science}

MIAAAS

We directly tested whether soil compaction restricted gas diffusion by experimentally

141 measuring ethylene's ability to move through compacted versus uncompacted soil. A $1 \mathrm{~cm}$ thick

142 soil column (connecting two air-filled chambers) was either left empty (control) or filled with

143 uncompacted soil $\left(1.1 \mathrm{~g} \mathrm{~cm}^{-3}\right)$ or compacted soil $\left(1.6 \mathrm{~g} \mathrm{~cm}^{-3}\right)$ (Fig. 3I and S25B). Ethylene was

144 injected into the upper chamber (an increase in pressure was avoided) and ethylene concentrations

145 were subsequently measured over time in the lower chamber until an equilibrium was reached

146 between the chambers. In agreement with gas diffusion simulations, ethylene levels rapidly

147 reached an equilibrium with the lower chamber in control conditions without soil resistance (Fig.

148 3I). Ethylene was also able to diffuse through uncompacted soil, albeit 10-50 times more slowly

149 than the empty control (Fig. 3I). In contrast, ethylene was unable to diffuse through compacted

150 soil, and was still undetectable in the lower chamber at 20 days (Fig. 3I). This result demonstrates

151 that soil compaction and the associated increase in soil moisture, due to less porosity, impacts

152 ethylene diffusion rates, consistent with our 'restricted gas diffusion' model. This much slower

153 ethylene diffusion in compacted soil results in an enhanced ethylene response in root cells. This

154 entrapped ethylene gas provides a fast and reliable signal for plants to interact with their

155 environment since nearly all roots produce ethylene under normoxic conditions (15).

156 Our results reveal how roots regulate growth responses to soil compaction. First, the

157 inhibition of root growth by compacted soils is triggered by ethylene signalling, rather than simply

158 by mechanical forces. Second, rather than using a dedicated mechano-perception mechanism, roots

159 appear to sense soil compaction through restricted diffusion of this gaseous signal from the plant

160 cells to the soil, causing ethylene to accumulate in root expansion zone cells, and inhibiting

161 elongation growth. Third, compaction and soil moisture status appear to impact root elongation,

162 not only because they control soil strength, but also through regulating ethylene diffusion. Fourth, 


\section{Science}

DAAAS

163 we propose that ethylene acts as an early warning signal for roots to avoid compacted soils (14)

164 providing a pathway for how breeders could select crops resilient to soil compaction.

165

166

\section{REFERENCES AND NOTES}

167 1. J. Correa, J. A. Postma, M. Watt, T. Wojciechowski, J. Exp. Bot. 70, 6019-6034 (2019).

168 2. B. D. Soane, P. S. Blackwell, J. W. Dickson, D. J. Painter, Soil Till. Res. 2, 3-36 (1982).

169 3. S. Mangalassery, S. Sjögersten, D. L. Sparkes, C. J. Sturrock, J. Craigon, S. J. Mooney, 170 Sci. Rep. 4, 1-8 (2014).

171 4. M. A. Hamza, W. K. Anderson, Soil Till. Res. 82, 121-145 (2005).

172 5. R. Horn, H. Domżżał, A. Słowińska-Jurkiewicz, C. Van Ouwerkerk, Soil Till. Res. 35, 23$173 \quad 36(1995)$.

6. T. Fujikawa, T. Miyazaki, Soil Sci. 170, 892-901 (2005).

7. A. Hussain, C. R. Black, I. B. Taylor, J. A. Roberts, Plant Physiol. 121, 1227-1237 (1999).

8. I. Potocka, J. Szymanowska-Pulka, Ann. Bot. 122, 711-723 (2018).

177

9. F. An et al., Plant Cell 22, 2384-2401 (2010).

178

10. D. A. Weits et al., Nature 569, 714-717 (2019).

179

11. S. Hartman et al., Nat. Commun. 10, 4020 (2019).

180

12. B. Ma et al., Mol. Plant. 6, 1830-1848 (2013).

182

13. J. M. Alonso, T. Hirayama, G. Roman, S. Nourizadeh, J. R. Ecker, Science 284, 21482152 (1999).

14. K. D. Montagu, J. P. Conroy, B. J. Atwell, J. Exp. Bot. 52, 2127-2133 (2001).

15. F. J. Romera, E. Alcantara, M. D. De la Guardia, Ann. Bot. 83, 51-55 (1999).

16. L. Lamaire, C. Deleu, E. L. Deunff. J. Exp. Bot. 64, 2725-2737 (2013).

17. P. Mehra P, B. K. Pandey, L. Verma, J. Giri J, Plant Cell Environ. 42, 1167-1179 (2019). 


\section{Science}

MIAAAS

187

188

18. W. Zhang, W. Gao, T. Ren, W. R. Whalley, Geoderma 368, 114276 (2020).

19. S. R. Tracy, K. R. Daly, C. J. Sturrock, N. M. J. Crout, S. J. Mooney, T. Roose, Water Resour. Res. 51, 1006-1022 (2015).

20. G. Huang et al., Nat. Commun. 9, 2346 (2018).

Acknowledgments: We thank R. Ashton for performing soil penetrometer assays, D. Weijers and D. Grierson for advice whilst drafting the manuscript, J. Dewick and P. Mehra for assisting with its submission and Otto van de Beek, Henkjan Siekman and Matthijs Krijnen of Jobshop, Utrecht University, for designing and creating the ethylene diffusion device. We thank Jinsong Zhang for providing rice oseil1/mhz6-1, osein2/mhz7-1 and proOsEIL1:GUS; Francesco Licausi for PCO1/2:GFP-GUS and Xin Zhou for Arabidopsis ein3eill lines.

Funding: This work was supported by Biotechnology and Biological Sciences Research Council (BB/G023972/1, $\quad$ BB/R013748/1, $\quad$ BB/L026848/1, $\quad$ BB/M018431/1, $\quad$ BB/PO16855/1, BB/M001806/1, BB/M012212, BB/P016855/1); ERC FUTUREROOTS Advanced grant 294729; B.K.P is supported by Future-Food Beacon and Challenge Grant-Royal Society (CHG\R1\170040). G.H. is supported by China Postdoctoral Science Foundation Project (2019M661486) and Shanghai Post-doctoral Excellent Program (2018063). R.B. is supported by BBSRC Discovery and Future Food Beacon Nottingham Research Fellowships. O.M. and M.K. is supported by Saclay Plant Sciences-SPS (ANR-17-EUR-0007) and Czech Foundation Agency (GJ20-25948Y), respectively. K.L. acknowledges support from the Swedish Foundation for Strategic Research (VINNOVA), the Swedish Research Council (VR) and the Knut and Alice Wallenberg foundation (KAW). 


\section{Science}

DAAAS

210 Author contributions: B.K.P., G.H., R.B., S.H., L.A.C.J.V., J.P.L., K.B., W.R.W., S.J.M., K.L.,

211 D.Z., and M.J.B. designed experiments; B.K.P., G.H., R.B., S.H., L.J., C.J.S. and M.K. performed

212 experiments. O.M. performed modelling. B.K.P., G.H., D.Z., and M.J.B. wrote the manuscript.

213 Competing interests: Authors declare no competing interests.

214 Data and materials availability: No restrictions are placed on materials, such as materials

215 transfer agreements. Details of all data, code, and materials used in the analysis are available in

216 the main text or the supplementary materials.

217 Supplementary Materials

218 Materials and Methods

219 Figs. S1 to S25

$220 \quad$ Movies S1 and S2

221 References (16-20)

222 MDAR Reproducibility Checklist

223

224

225

226

227

228

229

230

231

232 


\section{Science}

MIAAAS

233

234

235

236

237

238

239

240

241

242

243

244

245

246

247

248

249

250

251

252

253

254

255

256

257

258

259

260

261

262

263

264

265

266

267

268

269

270

271

272

273

274

275

276
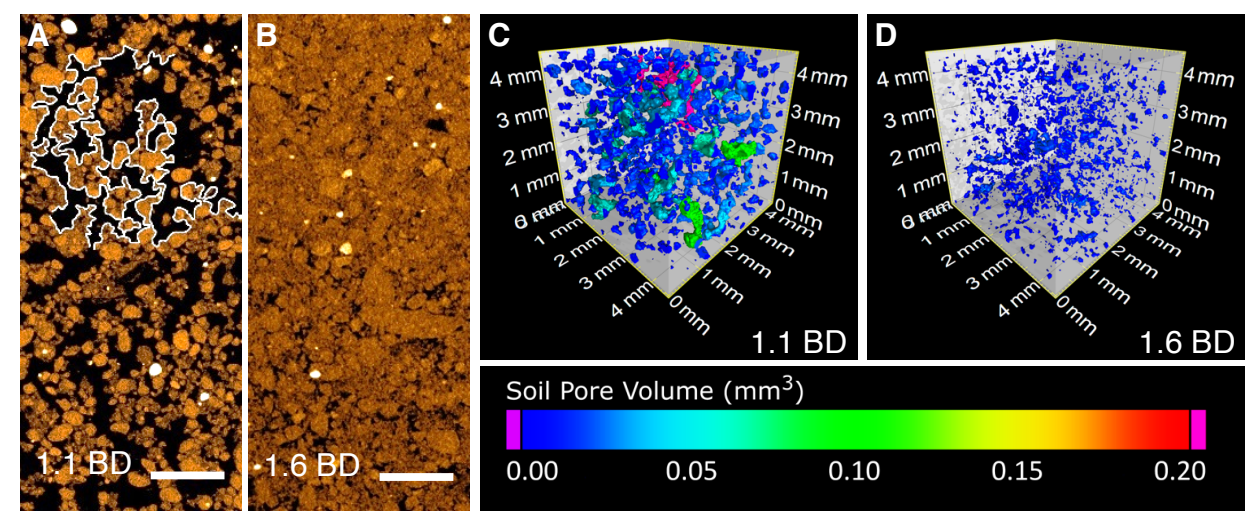

\section{Soil Pore Volume $\left(\mathrm{mm}^{3}\right)$}
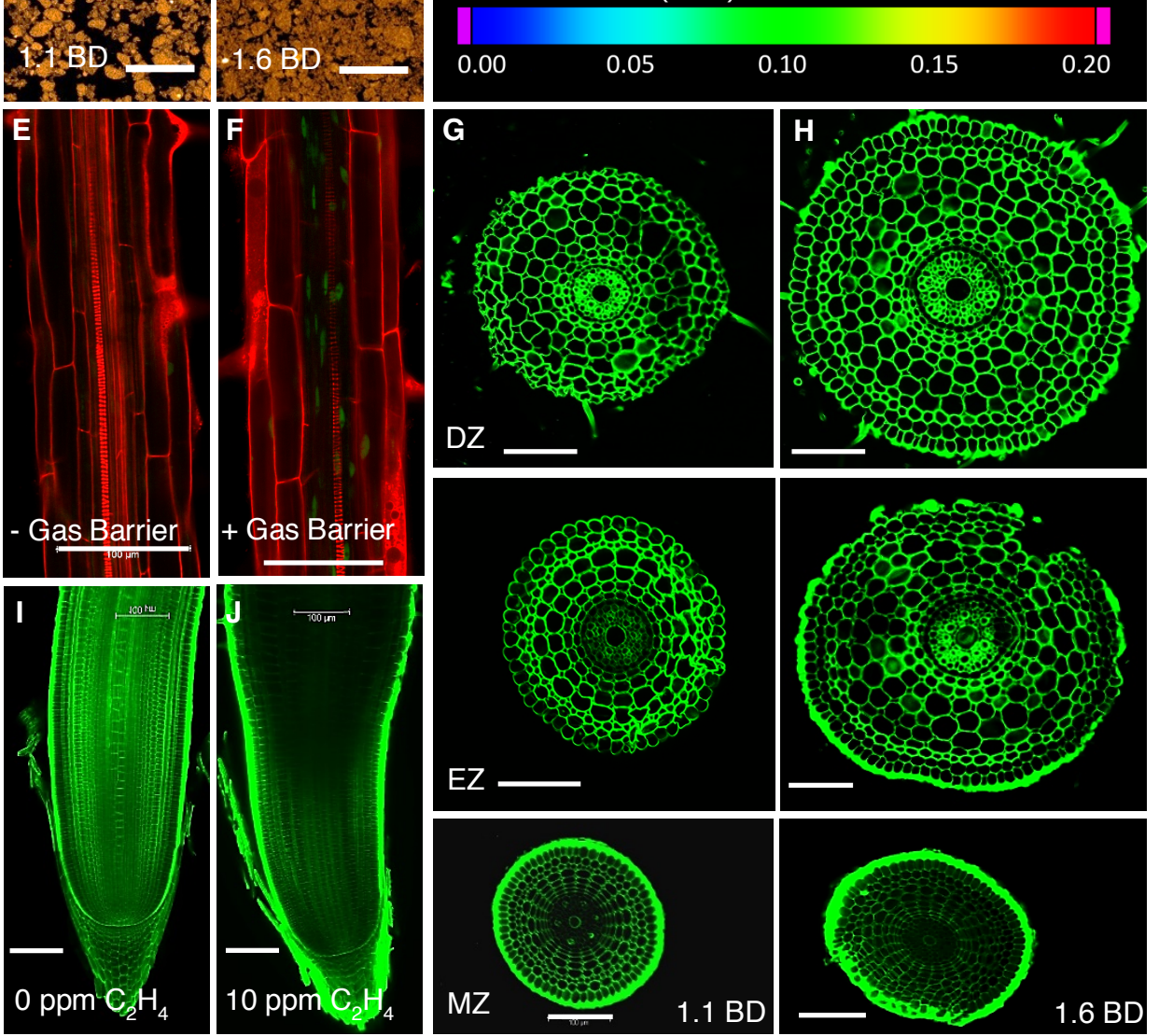

Fig. 1 Soil compaction reduces the larger pores and triggers root growth responses mimicking ethylene treatment. (A and B) CT images showing higher porosity (outlined in white) in uncompacted $\left(1.1 \mathrm{~g} \mathrm{~cm}^{-3}\right.$ bulk density [BD]) (A) versus compacted soil (1.6 BD) (B). (C and D) Representative 3D images of air-filled soil pores for a $100 \times 100 \times 100$ voxel region from $1.1 \mathrm{BD}$ (C) and 1.6 BD (D) soil cores. (E and F) Arabidopsis EIN3-GFP reporter exhibits elevated signal after covering root tip with high vacuum silicone grease (+Gas Barrier) for ten hours $(\mathrm{F})$ compared to control (-Gas Barrier) (E). ( $\mathbf{G}$ and $\mathbf{H})$ Confocal images of radial cross sections of rice primary roots through meristem (MZ), elongation (EZ) and differentiation (DZ) zones grown in 1.1 BD $(\mathrm{G})$ and 1.6 BD (H) soils. (I and $\mathbf{J})$ Compared to control roots (I), 10 ppm ethylene treated rice roots exhibit cortical cell expansion $(\mathrm{J})$, mimicking the effect of compacted soil conditions $(\mathrm{H})$. Bars, $1.25 \mathrm{~mm}$ in $\mathrm{A}$ and $\mathrm{B}$, and $100 \mu \mathrm{m}$ in $\mathrm{G}$ to $\mathrm{J}$. 


\section{Science}

MIAAAS
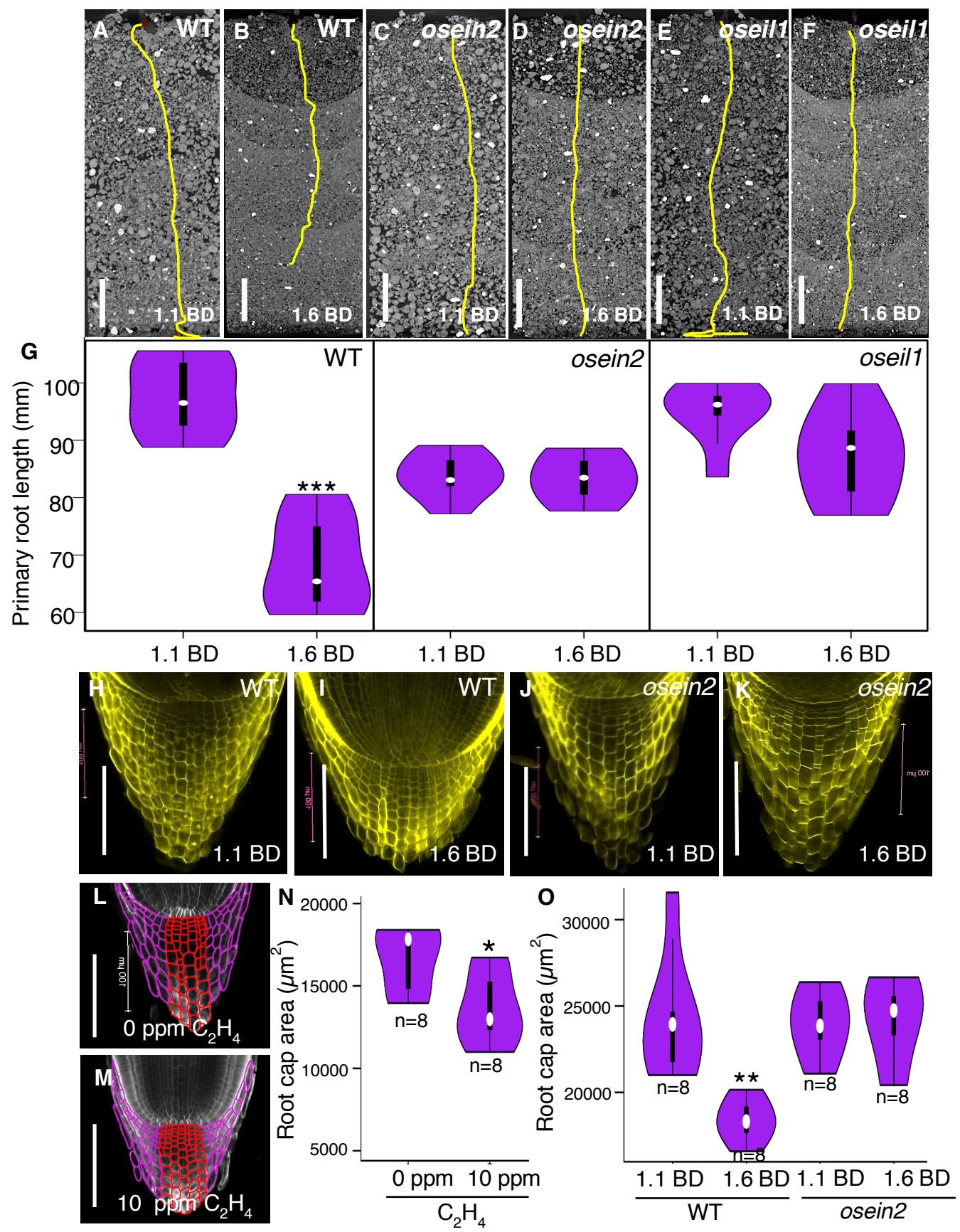

Fig. 2 Disrupting ethylene response in rice confers root growth resistance to compacted soil. (A to F) CT images of primary roots of WT (A and B), osein2 (C and D) and oseill (E and F) in $1.1 \mathrm{BD}(\mathrm{A}, \mathrm{C}$ and $\mathrm{E})$ vs $1.6 \mathrm{BD}(\mathrm{B}, \mathrm{D}$ and $\mathrm{F})$. (G) Violin plots of primary root length in uncompacted (1.1 BD) versus compacted (1.6 BD) conditions for WT (wildtype), osein2 and oseill rice seedlings. ( $\mathbf{H}$ to $\mathbf{K})$ Representative images showing root cap area in WT (H and I) and osein2 $(\mathrm{J}$ and $\mathrm{K})$ in $1.1 \mathrm{BD}(\mathrm{H}$ and $\mathrm{J})$ vs $1.6 \mathrm{BD}(\mathrm{I}$ and $\mathrm{K})$. ( $\mathbf{L}$ and $\mathbf{M})$ Ethylene treatment of WT roots showing reduction in root cap area (M versus L). (N) Violin plots showing reduction of root cap area after ethylene treatment. (O) Violin plots showing reduction of root cap area of WT but not osein2 when grown in 1.6 $\mathrm{BD}$ versus $1.1 \mathrm{BD}$. Columella cells are marked in red ( $\mathrm{L}$ and $\mathrm{M}) . *, * *$ and $* * *$ show $p$ value $\leq 0.05,0.001$ and 0.0001 , respectively determined using Student's $t$-test. Bars, $10 \mathrm{~mm}$ in $\mathrm{A}$ to $\mathrm{F}$ and $100 \mu \mathrm{m}$ in $\mathrm{H}$ to $\mathrm{M}$. 


\section{Science}

MlaAAS

323

324

325

326

327

328

329

330

331

332

333

334

335

336

337

338

339

340

341

342

343

344

345

346

347

348

349

350

351

352

353

354

355

356

357
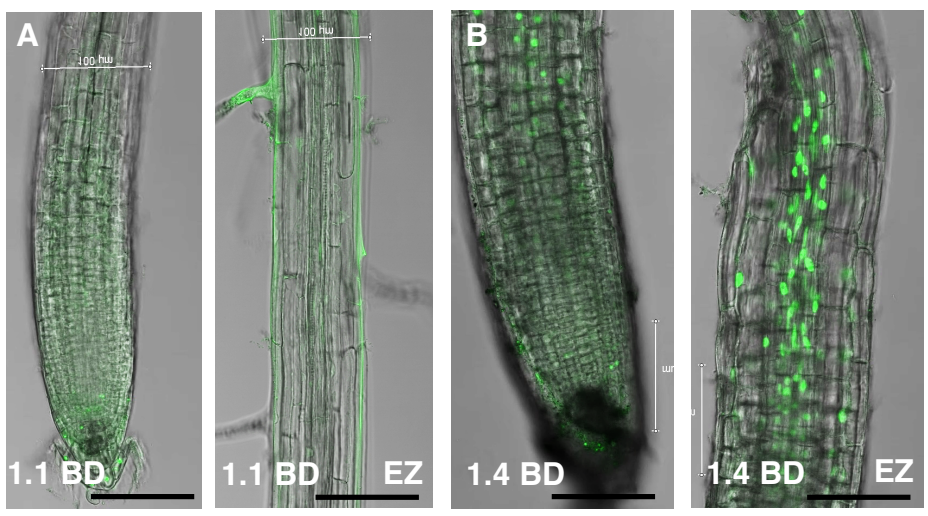

35S:EIN3-GFP/ein3eil1
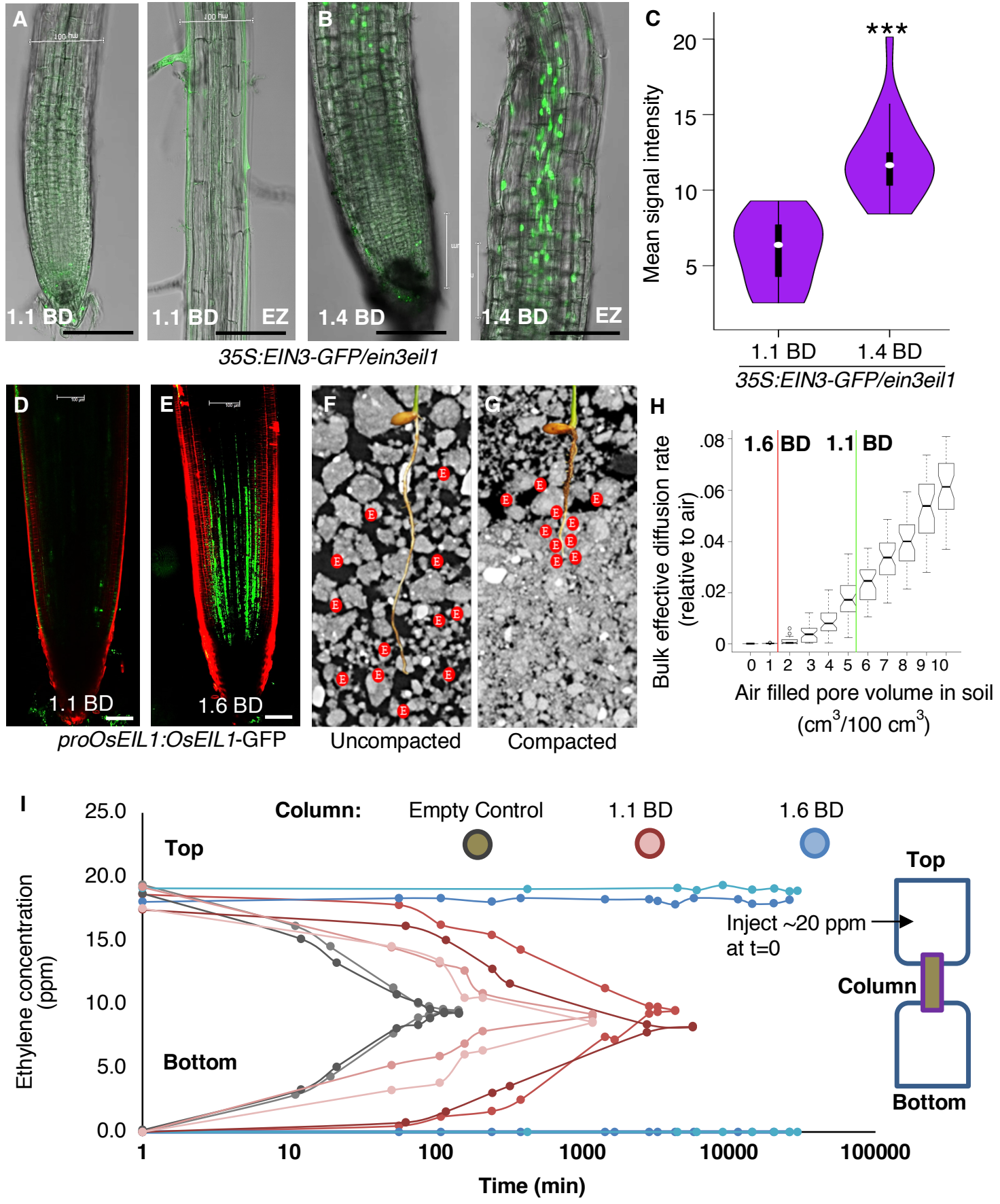

Fig. 3 Compacted soil reduces ethylene diffusion and enhances root ethylene signalling. (A and B) Arabidopsis ethylene reporter EIN3-GFP exhibits no nuclear GFP signal when grown in uncompacted soil (1.1 BD) (A), but is clearly detected in root EZ (elongation zone) cells when grown in compacted soil (1.4 BD) (B). (C) Violin plot of GFP signal in 1.1 BD versus 1.4 BD in EZ of 35S:EIN3-GFP/ein3eill. (D and E) Compared to 1.1 BD (D) rice OsEIL1-GFP based ethylene translational reporter exhibits elevated signal in compacted soil condition (1.6 BD) (E). (F and G) Schematic figures of ethylene diffusion (denoted by red circles) in uncompacted (F) versus $(\mathrm{G})$ compacted soil, illustrating preferential accumulation of ethylene around and in root tissues. (H) Model simulation showing rate of bulk diffusion of ethylene in soil pores in 


\section{Science}

МIAAAS

358 uncompacted (green line) and compacted soil (red line). \% air equates to $\mathrm{cm}^{3} / 100 \mathrm{~cm}^{3}$ (I) 359 Graphical representation of quantification of ethylene across 1.1 BD and 1.6 BD soil layers $(1 \mathrm{~cm})$.

$36020 \mathrm{ppm}$ of ethylene was injected in top chamber. Subsequently, ethylene diffusion in bottom 361 chamber was measured across empty, uncompacted (1.1 BD) and compacted (1.6 BD) soils using 362 GC-MS. *** shows $p \leq 0.0001$ evaluated using Student's $t$-test. 